\title{
Hamiltonian Polynomial Eigenvalue Problems
}

\author{
Mustapha Bassour1,2 \\ ${ }^{1}$ Faculté des Sciences Juridiques, Economiques et Sociales, Ait Melloul, Morocco \\ ${ }^{2}$ LISTI Laboratory ENSA, Ibn Zohr University, Agadir, Morocco \\ Email: m.bassour@uiz.ac.ma
}

How to cite this paper: Bassour, M. (2020) Hamiltonian Polynomial Eigenvalue Problems. Journal of Applied Mathematics and Physics, 8, 609-619. https://doi.org/10.4236/jamp.2020.84047

Received: January 8, 2020

Accepted: March 28, 2020

Published: March 31, 2020

Copyright $\odot 2020$ by author(s) and Scientific Research Publishing Inc. This work is licensed under the Creative Commons Attribution International License (CC BY 4.0).

http://creativecommons.org/licenses/by/4.0/

\begin{abstract}
We present in this paper a new method for solving polynomial eigenvalue problem. We give methods that decompose a skew-Hamiltonian matrix using Cholesky like-decomposition. We transform first the polynomial eigenvalue problem to an equivalent skew-Hamiltonian/Hamiltonian pencil. This process is known as linearization. Decomposition of the skew-Hamiltonian matrix is the fundamental step to convert a structured polynomial eigenvalue problem into a standard Hamiltonian eigenproblem. Numerical examples are given.
\end{abstract}

\section{Keywords}

Hamiltonian Matrix, Polynomial Eigenvalue Problem, Skew-Hamiltonian/Hamiltonian Pencil, Cholesky Like-Decomposition

\section{Introduction}

In this work we propose a numerical approach for solving the $k^{\text {th }}$ degree polynomial eigenvalue problem

$$
P(\lambda) v=\sum_{i=0}^{k} \lambda^{i} M_{i} v=0
$$

Problem (P) arises in many applications in science and engineering, ranging from the dynamical analysis of structural systems such as bridges and buildings to theories of elementary particles in atomic physics [1] [2]. It's also the most important task in the vibration analysis of buildings, machines, and vehicles [3]. We first transform our $k^{\text {th }}$ degree polynomial eigenvalue problem $(\mathbf{P})$ to an equivalent first-degree equation $(A-\lambda B) v=0$ commonly called pencil problem. In the case when matrices $M_{i}$ have symmetric/skew-symmetric structure, the problem (P) is transformed to a skew-Hamiltonian/Hamiltonian pencil [4]. The second step is to transform the skew-Hamiltonian/Hamiltonian pencil to a standard Hamiltonian eigenproblem $H v=\lambda v$ [5]. This transformation is ob- 
tained after decomposing $B$ as $R^{\mathrm{T}} J R$ where $R$ is a permuted triangular matrix. The Hamiltonian matrix $H$ is given by $J^{\mathrm{T}} R^{-\mathrm{T}} A R^{-1}$ where $J=\left(\begin{array}{cc}0 & I_{n} \\ -I_{n} & 0\end{array}\right)$.

It is known that any nonsingular skew-symmetric matrix has a decomposition of the form $B=R^{\mathrm{T}} J R$ [6]. The real matrix $M=J^{\mathrm{T}} B$ is skew-Hamiltonian and has the decomposition $J^{\mathrm{T}} B=R^{\mathrm{J}} R$ where $R$ has the form of a permuted triangular matrix. We give here a new proof for this result and different algorithms that compute the decomposition $M=R^{\mathrm{J}} R$.

\section{Preliminaries}

We give in this paragraph, new definitions and useful propositions.

Let $J=J_{2 n}=\left(\begin{array}{cc}0 & I_{n} \\ -I_{n} & 0\end{array}\right)$, where $I_{n}$ denotes the $n \times n$ identity matrix. We will use $J$ when the size is clear from the context. Recall that a matrix $M \in \mathbb{R}^{2 n \times 2 n}$ is skew-Hamiltonian if $M^{J}=M$, where the $J$-transpose of the matrix $M$ is defined by $M^{J}=J^{T} M^{\mathrm{T}} J$. Likewise, a Hamiltonian matrix $H$ is written as $\left(\begin{array}{cc}E & G \\ F & -E^{\mathrm{T}}\end{array}\right)$ where $E, G$ and $T \in \mathbb{R}^{n \times n}$ with $G^{\mathrm{T}}=G$ and $F^{\mathrm{T}}=F$. We have $H^{J}=-H$. More general, the $J$-transpose of the rectangular $2 p$-by- $2 q$ matrix $N$ is defined by $2 q$-by-2p matrix $N^{J}=J_{2 q}^{\mathrm{T}} N^{\mathrm{T}} J_{2 p}$.

The set $\left(E_{i}\right)_{1 \leq i \leq n}$ where $E_{i}=\left[\begin{array}{ll}e_{i} & e_{n+i}\end{array}\right]$ with $e_{i}$ is denoting the $i$-th unit vector of length $2 n$, satisfies $E_{i} J_{2}=J_{2 n} E_{i}, E_{i}^{J}=E_{i}^{\mathrm{T}}$ and $E_{i}^{\mathrm{T}} E_{j}=\delta_{i j} I_{2}$ where $E_{i}^{J}=J_{2}^{\mathrm{T}} E_{i}^{\mathrm{T}} J_{2 n}$ and $\delta_{i j}= \begin{cases}1 & \text { if } i=j \\ 0 & \text { if } i \neq j\end{cases}$

Let $U=\left[u_{1}, u_{2}\right] \in R^{2 n \times 2}$ where $u_{1}=\sum_{i=1}^{2 n} u_{i}^{1} e_{i}$ and $u_{2}=\sum_{j=1}^{2 n} u_{j}^{2} e_{j}$. Then $U$ is written in a unique way as linear combination of $\left(E_{i}\right)_{1 \leq i \leq n}$ on $\mathbb{R}^{2 \times 2}, U=\sum_{i=1}^{n} E_{i} M_{i}$ where $M_{i}=\left(\begin{array}{cc}u_{i}^{1} & u_{i}^{2} \\ u_{n+i}^{1} & u_{n+i}^{2}\end{array}\right)$. Let $M \in \mathbb{R}^{2 n \times 2 n}$ be a $2 n$-by- $2 n$ real matrix. Then $M$ is written as $M=\sum_{i=1}^{n} \sum_{j=1}^{n} E_{i} M_{i j} E_{j}^{\mathrm{T}}$ where $M_{i j}=\left(\begin{array}{cc}m_{i j} & m_{i, n+j} \\ m_{n+i, j} & m_{n+i, n+j}\end{array}\right)$.

Definition 2.1. The $2 n$-by- $2 n$ real matrix $L=\sum_{i=1}^{n} \sum_{j=1}^{n} E_{i} L_{i j} E_{j}^{\mathrm{T}}$ is called lower $J$-triangular if $L_{i j}=0_{2 \times 2}$ for $j>i$ and $L_{i i}=\left(\begin{array}{cc}* & 0 \\ * & *\end{array}\right)$, (i.e., $\left.L=\sum_{i=1}^{n} \sum_{j=1}^{i} E_{i} L_{i j} E_{j}^{\mathrm{T}}\right)$.

Definition 2.2. The $2 n$-by- $2 n$ real matrix $U=\sum_{i=1}^{n} \sum_{j=1}^{n} E_{i} U_{i j} E_{j}^{\mathrm{T}}$ is called upper $J$-triangular if $U_{i j}=0_{2 \times 2}$ for $i>j$ and $U_{i i}=\left(\begin{array}{ll}* & * \\ 0 & *\end{array}\right)$, (i.e., $\left.U=\sum_{i=1}^{n} \sum_{j=i}^{n} E_{i} U_{i j} E_{j}^{\mathrm{T}}\right)$.

Proposition 2.1. Let $M$ and $N$ be two upper $J$-triangular (respectively, lower $J$-triangular) $2 n$-by- $2 n$ real matrix. The product $P=M N$ remain as upper 
$J$-triangular (respectively, as lower $J$-triangular).

Proof. Let $M=\sum_{i=1}^{n} \sum_{j=i}^{n} E_{i} M_{i j} E_{j}^{\mathrm{T}}$ and $N=\sum_{i=1}^{n} \sum_{j=i}^{n} E_{i} N_{i j} E_{j}^{\mathrm{T}}$ two upper $J$-triangular $2 n$-by- $2 n$ real matrix. The matrix product of $M$ and $N$ is obtained by

$$
P=\sum_{i=1}^{n} \sum_{j=i}^{n} E_{i} M_{i j} \sum_{k=j}^{n} N_{j k} E_{k}^{\mathrm{T}}=\sum_{i=1}^{n} \sum_{k=i}^{n} E_{i} \underbrace{\left(\sum_{j=i}^{k} M_{i j} N_{j k}\right)}_{P_{i k}} E_{k}^{\mathrm{T}} .
$$

That proves $P=M N$ remain as upper $J$-triangular. (similarly, when $M$ and $N$ are lower $J$-triangular).

Definition 2.3. $U=\left[u_{1}, u_{2}\right] \in \mathbb{R}^{2 n \times 2}$ is called $J$-isotropic if $U^{J} U=\left(u_{1}^{\mathrm{T}} J u_{2}\right) I_{2}=0_{2}$.

Proposition 2.2. The inverse of a regular upper $J$-triangular $2 n$-by- $2 n$ real matrix (respectively, lower $J$-triangular) is also upper $J$-triangular (respectively, also lower $J$-triangular).

Proof. Let $U=\sum_{i=1}^{n} \sum_{j=i}^{n} E_{i} U_{i j} E_{j}^{\mathrm{T}}$ an upper $J$-triangular $2 n$-by- $2 n$ real matrix. The following proposition $\left(P_{k}\right): U^{-1} E_{k}=\sum_{l=1}^{k} E_{l} C_{l}$, where $\left(C_{l}\right)_{1 \leq l \leq k}$ are 2-by-2 real matrix, holds for $k=1$. Suppose $\left(P_{i}\right)_{1 \leq i \leq k}$ are true for $1 \leq i \leq k,(k<n)$. For $i=k+1$, we have $U^{-1} E_{k+1}=\sum_{i=1}^{k+1} E_{i} U_{i k+1}$, therefore

$$
\begin{aligned}
\underbrace{U^{-1}\left(U E_{k+1}\right)}_{E_{k+1}} & =U^{-1}\left(\sum_{i=1}^{k} E_{i} U_{i k+1}+E_{k+1} U_{k+1 k+1}\right) \\
& =\sum_{i=1}^{k} U^{-1} E_{i} U_{i k+1}+U^{-1} E_{k+1} U_{k+1 k+1}
\end{aligned}
$$

Since $U_{k+1, k+1}=\left(\begin{array}{cc}u_{k+1, k+1} & * \\ 0 & u_{n+k+1, n+k+1}\end{array}\right)$ is invertible and by using recurrence hypothesis, then

$$
\begin{aligned}
U^{-1} E_{k+1} & =E_{k+1} U_{k+1 k+1}^{-1}-\sum_{i=1}^{k} U^{-1} E_{i}\left(U_{i k+1} U_{k+1 k+1}^{-1}\right) \\
& =E_{k+1} U_{k+1 k+1}^{-1}-\sum_{i=1}^{k}\left(\sum_{j=1}^{i} E_{j} C_{j}\right)\left(U_{i k+1} U_{k+1 k+1}^{-1}\right) \\
& =E_{k+1} U_{k+1 k+1}^{-1}-\sum_{j=1}^{k} E_{j} C_{j}\left(\sum_{i=j}^{k} U_{i k+1} U_{k+1 k+1}^{-1}\right) \\
& =\sum_{l=1}^{k+1} E_{l} G_{l}
\end{aligned}
$$

with $G_{k+1, k+1}=U_{k+1, k+1}^{-1}=\left(\begin{array}{cc}\frac{1}{u_{k+1, k+1}} & * \\ 0 & \frac{1}{u_{n+k+1, n+k+1}}\end{array}\right)$. 


\section{Cholesky Like-Decomposition for Skew-Hamiltonian Matrix}

In this section, we study different ways to compute $R^{J} R$ decomposition of a real skew-Hamiltonian matrix $M \in \mathbb{R}^{2 n \times 2 n}$. We began first by giving some interesting theoretical results.

\subsection{Definition and Theoretical Results}

Definition 3.1. The $2 n$-by- $2 n$ real skew-Hamiltonian matrix $M$ is called $J$-definite if $U^{J} M U=\alpha I_{2}$ with $\alpha \neq 0$ for every non $J$-isotropic $U=\left[u_{1}, u_{2}\right] \in \mathbb{R}^{2 n \times 2} \quad\left(\right.$ i.e., $\left.u_{1}^{\mathrm{T}} J u_{2} \neq 0\right)$.

Remark 3.1 For $U=\left[u_{1}, u_{2}\right] \in \mathbb{R}^{2 n \times 2}$ and a $2 n$-by-2n real skew-Hamiltonian $\operatorname{matrix} M, U^{J} M U=\left(\begin{array}{cc}-u_{2}^{\mathrm{T} J M u_{1}} & -\underbrace{u_{2}^{\mathrm{T}} J M u_{2}}_{0} \\ \underbrace{u_{1}^{\mathrm{T}} J M u_{1}}_{0} & u_{1}^{\mathrm{T}} J M u_{2}\end{array}\right)=\alpha I_{2} \quad$ with $\alpha=u_{1}^{\mathrm{T}} J M u_{2}$.

Lemma 3.1. If $M$ is a $2 n$-by- $2 n$ real skew-Hamiltonian and $J$-definite matrix, then $M$ is invertible.

Proof. If not, there exists $v \in \mathbb{R}^{2 n}$ such that $M v=0_{\mathbb{R}^{2 n}}$. Let $u \in \mathbb{R}^{2 n}$ that verify $U=[u, v] \in \mathbb{R}^{2 n \times 2}$ non $J$-isotropic (i.e., $u^{\mathrm{T}} J v \neq 0$ ). Since $M v=0_{\mathbb{R}^{2 n}}$, then $U^{J} M U=\underbrace{\left(u^{\mathrm{T}} J M v\right)}_{0} I_{2}=0_{2}$ which is contradictory with the hypothesis.

Theorem 3.2. If $M$ is a $2 n$-by- $2 n$ real skew-Hamiltonian, $J$-definite matrix, then $M$ has an $L U J$-factorization.

Proof. Let $W \in \mathbb{R}^{2 k \times 2} \quad(1 \leq k \leq n)$ be non $J$-isotropic. Suppose that $W=\sum_{i=1}^{k} E_{i} C_{i}$ where $C_{i} \in \mathbb{R}^{2 \times 2}$. We construct an $U \in \mathbb{R}^{2 n \times 2}$ such that $U=\sum_{i=1}^{n} E_{i} C_{i} \quad$ where $C_{i}=0_{2 \times 2}$ for $i>k$. We have, $U^{J} M U=W^{J} M^{[k, k]} W$ where $M^{[k, k]}=\sum_{i=1}^{k} \sum_{j=1}^{k} E_{i} M_{i j} E_{j}^{\mathrm{T}}$ as defined in theorem 2.2 given above. Then $2 k$-by-2 $k$ matrix $M^{[k, k]}$ remains skew-Hamiltonian and $J$-definite and then invertible.

Corollary 3.3. If $M=L U$ is the $L U J$-factorization of the real $2 n$-by- $2 n$ skew-Hamiltonian, $J$-definite matrix $M$, then $M$ has an $M=L \Delta L^{J}$ where $\Delta=\sum_{i=1}^{n} E_{i}\left(\begin{array}{cc}u_{i i} & 0 \\ 0 & u_{i i}\end{array}\right) E_{i}^{\mathrm{T}}$ (here $u_{i i}$ is the $i$-th diagonal entry of $U$ ).

Proof. Since the matrix $M$ is skew-Hamiltonian, then by taking $U=\Delta N$ we obtain

$$
M=L U=U^{J} L^{J}=N^{J} \Delta^{J} L^{J}=N^{J}(L \Delta)^{J}
$$

$M=N^{J}(L \Delta)^{J}$ is nothing but the $L U \quad J$-factorization of $M$. Indeed, $N^{J}$ is lower $J$-triangular with 1 in diagonal and $(L \Delta)^{J}$ is upper $J$-triangular. Thus, from the uniqueness of the $L U J$-factorization, it follows that $N^{J}=L$. 
Theorem 3.4. Let $M$ be a $2 n$-by- $2 n$ real skew-Hamiltonian $J$-definite matrix, then $M$ has a Cholesky $J$-factorization $M=N N^{J}$ where $N$ is lower $J$-triangular and in addition the $N_{i i}=\left(\begin{array}{cc}n_{i, i} & 0 \\ 0 & n_{n+i, n+i}\end{array}\right)$ are diagonal.

Proof. We proceed by induction on $n$. For $n=1$, the real 2-by-2 skew-Hamiltonian $J$-definite matrix $M=m_{11} I_{2}$ where $m_{11} \neq 0$. If we set $N=\left(\begin{array}{cc}\sqrt{\left|m_{11}\right|} & 0 \\ 0 & \operatorname{sign}\left(m_{11}\right) \sqrt{\left|m_{11}\right|}\end{array}\right)$, the theorem holds trivially.

Let's now $n \geq 2$. Since $M$ is skew-Hamiltonian and $J$-definite, then $E_{1}^{J} M E_{1}=m_{11} I_{2} \neq 0_{2}$. We can write

$$
\begin{aligned}
M= & \sum_{i=1}^{n} \sum_{j=1}^{n} E_{i} M_{i j} E_{j}^{\mathrm{T}} \\
= & E_{1} M_{11} E_{1}^{\mathrm{T}}+\sum_{i=2}^{n} E_{i} M_{i 1} E_{1}^{\mathrm{T}}+\sum_{i=2}^{n} E_{1} M_{i 1}^{J} E_{i}^{\mathrm{T}} \\
& +\sum_{i=2}^{n} \sum_{j=2}^{n} E_{i} M_{i j} E_{j}^{\mathrm{T}} .
\end{aligned}
$$

We set $W=\sum_{i=2}^{n} E_{i} M_{i 1} E_{1}^{\mathrm{T}}$ and $B=\sum_{i=2}^{n} \sum_{j=2}^{n} E_{i} M_{i j} E_{j}^{\mathrm{T}}$. The $J$-transpose of $W$ is given by $W^{J}=\sum_{i=2}^{n} E_{1} M_{i 1}^{J} E_{i}^{\mathrm{T}}$. Let $K=\left(\begin{array}{cc}\sqrt{\left|m_{11}\right|} & 0 \\ 0 & \operatorname{sign}\left(m_{11}\right) \sqrt{\left|m_{11}\right|}\end{array}\right)$, $B_{1}=E_{1} K E_{1}^{\mathrm{T}}+\sum_{i=2}^{n} E_{i} M_{i 1} K^{-J} E_{1}^{\mathrm{T}}+\sum_{i=2}^{n} E_{i} E_{i}^{\mathrm{T}}$ and $A_{1}=E_{1} E_{1}^{\mathrm{T}}+B-\frac{1}{m_{11}} W W^{J} . \mathrm{We}$ calculate $B_{1} A_{1}=E_{1} K E_{1}^{\mathrm{T}}+\sum_{i=2}^{n} E_{i} M_{i 1} K^{-J} E_{1}^{\mathrm{T}}+B-\frac{1}{m_{11}} W W^{J}$. The $J$-transpose of $B_{1}$ is given by $B_{1}^{J}=E_{1} K^{J} E_{1}^{\mathrm{T}}+\sum_{i=2}^{n} E_{1} K^{-1} M_{i 1}^{J} E_{i}^{\mathrm{T}}+\sum_{i=2}^{n} E_{i} E_{i}^{\mathrm{T}}$. Finally, we obtain

$$
\begin{aligned}
B_{1} A_{1} B_{1}^{J}= & m_{11} E_{1} E_{1}^{\mathrm{T}}+\sum_{i=2}^{n} E_{1} \underbrace{\left(K K^{-1}\right)}_{I_{2}} M_{i 1}^{J} E_{i}^{\mathrm{T}}+\sum_{i=2}^{n} E_{i} M_{i 1} \underbrace{\left(K^{-J} K^{J}\right)}_{I_{2}} E_{1}^{\mathrm{T}} \\
& +\sum_{i=2}^{n} \sum_{j=2}^{n} E_{i} M_{i 1} \underbrace{\left(K^{-J} K^{-1}\right)}_{\frac{1}{m_{11}} I_{2}} M_{j 1}^{J} E_{j}^{\mathrm{T}}+B-\frac{1}{m_{11}} W W^{J}
\end{aligned}
$$

Since $W W^{J}=\sum_{i=2}^{n} \sum_{j=2}^{n} E_{i} M_{i 1} M_{j 1}^{J} E_{j}^{\mathrm{T}}$, then $B_{1} A_{1} B_{1}^{J}=M$. By induction $B-\frac{1}{m_{11}} W W^{J}=L L^{J}$, where $L=\sum_{i=2}^{n} \sum_{j=2}^{i} E_{i} L_{i j} E_{j}^{\mathrm{T}}$ where $L$ is $2(n-1)-$ by- $2(n-1)$ lower $J$-triangular matrix and in addition the $L_{i i}=\left(\begin{array}{cc}l_{i, i} & 0 \\ 0 & l_{n+i, n+i}\end{array}\right)$ are diagonal for $i=2, \cdots, n$. Therefore, if we let $G=E_{1} E_{1}^{\mathrm{T}}+L L^{J}$, we obtain $G G^{J}=E_{1} E_{1}^{\mathrm{T}}+L L^{J}=A_{1}$ and then finally 


$$
M=B_{1} A_{1} B_{1}^{J}=B_{1}\left(G G^{J}\right) B_{1}^{J}=\left(B_{1} G\right)\left(B_{1} G\right)^{J}=N N^{J} .
$$

Since $B_{1}$ and $G$ are lower $J$-triangular, then $N=B_{1} G$ remain lower $J$-triangular and verify $N_{i i}=\left(\begin{array}{cc}n_{i, i} & 0 \\ 0 & n_{n+i, n+i}\end{array}\right)$ are diagonal.

\subsection{Method 1}

We construct an algorithm that gives decomposition $R^{J} R$ of skew-Hamiltonian matrices via a $L U \quad J$-decomposition.

Proposition 3.5. Let $M$ is a $2 n$-by- $2 n$ real skew-Hamiltonian, $J$-definite matrix. If $M=L U$ its $L U \quad J$-factorization, then $R=(L D)^{J}$ where $D$ is a diagonal matrix defined by

$$
D=\sum_{i=1}^{n} E_{i}\left(\begin{array}{cc}
\sqrt{\operatorname{sign}\left(u_{i i}\right) u_{i i}} & 0 \\
0 & \operatorname{sign}\left(u_{i i}\right) \sqrt{\operatorname{sign}\left(u_{i i}\right) u_{i i}}
\end{array}\right) E_{i}^{\mathrm{T}}
$$

(here $u_{i i}$ is the $i$-th diagonal entry of $U$ ) is lower $J$-triangular and verify $M=R^{J} R$.

Proof. By corollary 3.3, $M=L \Delta L^{J}$. Since $\Delta=D D^{J}=\sum_{i=1}^{n} E_{i}\left(\begin{array}{cc}u_{i i} & 0 \\ 0 & u_{i i}\end{array}\right) E_{i}^{T}$ where $D$ is as given above, then $R=(L D)^{J}$ is lower $J$-triangular and $R^{J} R=\underbrace{L D^{J}}_{\Delta} L^{J}=M$. From the $J$-decomposition $M=L U$ given by algorithms in section, we set

$$
D=\sum_{i=1}^{n} E_{i}\left(\begin{array}{cc}
\sqrt{\operatorname{sign}\left(u_{i i}\right) u_{i i}} & 0 \\
0 & \operatorname{sign}\left(u_{i i}\right) \sqrt{\operatorname{sign}\left(u_{i i}\right) u_{i i}}
\end{array}\right) E_{i}^{\mathrm{T}}
$$

where $u_{i i}=e_{i}^{\mathrm{T}} U e_{i}$. We have $M=R^{J} R$ where $R=(L D)^{J}$.

\subsection{Method 2}

We study now a method that constructs decomposition $R^{J} R$ of skew-Hamiltonian $J$-definite matrices.

Let $M \in \mathbb{R}^{2 n \times 2 n}$ be a skew-Hamiltonian $J$-definite matrix.

$$
M=\sum_{i=1}^{n} \sum_{j=1}^{n} E_{i} M_{i j} E_{j}^{\mathrm{T}}
$$

with $\quad M_{i j}=\left(\begin{array}{cc}m_{i, j} & m_{i, n+j} \\ m_{n+i, j} & m_{n+i, n+j}\end{array}\right)$ and $E_{i}=\left[\begin{array}{ll}e_{i} & e_{n+i}\end{array}\right] \in \mathbb{R}^{2 n \times 2}$. Let $M=L L^{J} \quad$ where $L$ is lower $J$-triangular that verify $L_{i i}=\left(\begin{array}{cc}l_{i, i} & 0 \\ 0 & l_{n+i, n+i}\end{array}\right)$. The existence of $L$ is guaranteed by theorem 4.4

$$
L=\sum_{i=1}^{n} \sum_{j=i}^{n} E_{i} L_{i j} E_{j}^{\mathrm{T}}
$$

Since 


$$
\begin{aligned}
L L^{J} & =\left(\sum_{i=1}^{n} \sum_{j=1}^{i} E_{i} L_{i j} E_{j}^{\mathrm{T}}\right)\left(\sum_{k=1}^{n} \sum_{s=1}^{k} E_{s} L_{k s}^{J} E_{k}^{\mathrm{T}}\right)=\sum_{i=1}^{n} \sum_{s=1}^{i} \sum_{k=s}^{n} E_{i} L_{i s} L_{k s}^{J} E_{k}^{\mathrm{T}} \\
& =\sum_{i=1}^{n} \sum_{j=1}^{n} E_{i}\left[\sum_{s=1}^{i} L_{i s} L_{j s}^{J}\right] E_{j}^{\mathrm{T}}=\sum_{i=1}^{n} \sum_{j=1}^{n} E_{i} M_{i j} E_{j}^{\mathrm{T}}
\end{aligned}
$$

then

$$
M_{i j}=\sum_{s=1}^{\min (i, j)} L_{i s} L_{j s}^{J}
$$

If $i=1, j=1$ then $s=1$. We obtain $L_{11} L_{11}^{J}=M_{11}$. Since $L_{11} L_{11}^{J}=\operatorname{det}\left(L_{11}\right) I_{2}$ and $M_{11}=m_{11} I_{2}$, then $l_{11} \times l_{n+1 n+1}=m_{11}$, then

$$
\left\{\begin{array}{l}
l_{11}=\sqrt{\operatorname{sign}\left(m_{11}\right) m_{11}} \\
l_{n+1 n+1}=\operatorname{sign}\left(m_{11}\right) \sqrt{\operatorname{sign}\left(m_{11}\right) m_{11}}
\end{array}\right.
$$

And for $i=2,3, \cdots, n, \quad M_{i 1}=L_{i 1} L_{11}^{J}$. Multiplying on the right by $L_{11}$, we obtain

$$
M_{i 1} L_{11}=L_{i 1} L_{11}^{J} L_{11}=\operatorname{det}\left(L_{11}\right) L_{i 1}
$$

Thus

$$
L_{i 1}=\frac{M_{i 1} L_{11}}{\operatorname{det}\left(L_{11}\right)}=M_{i 1}\left(\begin{array}{cc}
\frac{\sqrt{\operatorname{sign}\left(m_{11}\right) m_{11}}}{m_{11}} & 0 \\
0 & \frac{\operatorname{sign}\left(m_{11}\right) \sqrt{\operatorname{sign}\left(m_{11}\right) m_{11}}}{m_{11}}
\end{array}\right)
$$

Since

$$
M_{22}=L_{21} L_{21}^{J}+L_{22} L_{22}^{J}=\operatorname{det}\left(L_{21}\right) I_{2}+\operatorname{det}\left(L_{22}\right) I_{2}
$$

then,

$$
\operatorname{det}\left(L_{22}\right) I_{2}=M_{22}-\operatorname{det}\left(L_{21}\right) I_{2}=\left(m_{22}-\operatorname{det}\left(L_{21}\right)\right) I_{2}
$$

Since $\operatorname{det}\left(L_{22}\right)=l_{22} \times l_{n+2 n+2}$, then

If we set $\alpha_{2}=m_{22}-\operatorname{det}\left(L_{21}\right)$, then we obtain

$$
L_{22}=\left(\begin{array}{cc}
\sqrt{\operatorname{sign}\left(\alpha_{2}\right) \alpha_{2}} & 0 \\
0 & \operatorname{sign}\left(\alpha_{2}\right) \sqrt{\operatorname{sign}\left(\alpha_{2}\right) \alpha_{2}}
\end{array}\right) .
$$

However $\quad M_{i 2}=L_{i 1} L_{21}^{J}+L_{i 2} L_{22}^{J} \quad$ for $i=2,3, \cdots, n$, then $L_{i 2} L_{22}^{J}=M_{i 2}-L_{i 1} L_{21}^{J}$. Multiplying on the right by $L_{22}$ we find $L_{i 2} L_{22}^{J} L_{22}=\left(M_{i 2}-L_{i 1} L_{21}^{J}\right) L_{22}$ and finally $L_{i 2}=\frac{\left(M_{i 2}-L_{i 1} L_{21}^{J}\right) L_{22}}{\operatorname{det}\left(L_{22}\right)}$.

The method yield the following algorithm.

\section{Algorithm:}

for $j=1,2, \cdots, n$

$$
\alpha_{j}=m_{j j}-\sum_{s=1}^{j-1} \operatorname{det}\left(L_{j s}\right)
$$




$$
L_{j j}=\sqrt{\operatorname{sign}\left(\alpha_{j}\right) \alpha_{j}}\left(\begin{array}{cc}
1 & 0 \\
0 & \operatorname{sign}\left(\alpha_{j}\right)
\end{array}\right)
$$

for $i=j+1, j+2, \cdots, n$

$$
L_{i j}=\frac{\left(M_{i j}-\sum_{s=1}^{j-1} L_{i s} L_{j s}^{J}\right) L_{j j}}{\operatorname{det}\left(L_{j j}\right)}
$$

\section{Polynomial Eigenvalue Problems}

Many applications give rise to structured matrix polynomial eigenvalue problems

$$
P(\lambda) \nu=\sum_{i=0}^{k} \lambda^{i} M_{i} v=0
$$

The numerical solution of this polynomial eigenvalue problem is one of the most important tasks in the vibration analysis of buildings, machines and vehicles [7]. In many applications, the coefficient matrices have particular structure and it is important that numerical methods respect this structure. A popular approach for solving the polynomial eigenvalue problem $P(\lambda) v=0$ is to linearize to produce a generalized eigenproblem $(A-\lambda B) v=0$ [8].

Theorem 4.1. [9] Consider the polynomial eigenvalue problem $P(\lambda) v=0$ with either $M_{i}^{\mathrm{T}}=(-1)^{i} M_{i}$ or $M_{i}^{\mathrm{T}}=(-1)^{i+1} M_{i}$ and with $M_{k}$ nonsingular. Then solving problem $P(\lambda) v=0$ is equivalent to solve $(A-\lambda B) v=0$ where

$$
A=\left(\begin{array}{ccccccc}
-M_{0} & 0 & 0 & 0 & \cdots & \cdots & 0 \\
0 & -M_{2} & -M_{3} & -M_{4} & \cdots & \cdots & -M_{k} \\
0 & M_{3} & M_{4} & & & & 0 \\
0 & -M_{4} & & & & & 0 \\
\vdots & \vdots & & & & & \vdots \\
\vdots & \vdots & & & & & \vdots \\
0 & \pm M_{k} & 0 & 0 & \cdots & \cdots & 0
\end{array}\right)
$$

and

$$
B=\left(\begin{array}{ccccccc}
M_{1} & M_{2} & M_{3} & \cdots & \cdots & M_{k-1} & M_{k} \\
-M_{2} & -M_{3} & -M_{4} & \cdots & \cdots & -M_{k} & 0 \\
M_{3} & M_{4} & & & & & 0 \\
-M_{4} & & & & & & 0 \\
\vdots & & & & & & \vdots \\
\vdots & & & & & & \vdots \\
\pm M_{k} & 0 & 0 & 0 & \cdots & \cdots & 0
\end{array}\right)
$$

We draw from this theorem that the polynomial eigenvalue problem $(\mathrm{P})$ can be reduced to an eigenvalue pencil problem $A v=\lambda B v$ where $A$ is symmetric and $B$ is skew-symmetric. The second step is to transform the skew-symmetric/ symmetric pencil to a standard Hamiltonian eigenproblem $H v=\lambda v$ by decomposing the skew-Hamiltonian matrix $J B$ as $R^{J} R$. The Hamiltonian ma- 
trix $H$ is then obtained by $J^{\mathrm{T}} R^{-\mathrm{T}} A R^{-1}$. Eigenvalue problems of this type arise property that all eigenvalues appear in quadruples $(\lambda,-\lambda, \bar{\lambda},-\bar{\lambda})$, the spectrum is symmetric with respect to the real and imaginary axes.

\section{Numerical Examples}

We present computed eigenvalues that solve the $k^{\text {th }}$ degree polynomial eigenvalue problem $P(\lambda) v=\sum_{p=0}^{k} \lambda^{p} M_{p} v=0$ of dimension $n \times n$ which is transforming to a standard eigenvalue problem of dimension $k n \times k n$. We also compute the error consisting in

$$
\text { err }=\sum_{i=1}^{k n}\left\|P\left(\lambda_{i}\right) v^{(i)}\right\|_{2}
$$

\section{Example 1. [9]}

Let us consider a quartic eigenvalue problem of the form $\left(\lambda^{4} M_{4}+\lambda^{3} M_{3}+\lambda^{2} M_{2}+\lambda M_{1}+M_{0}\right) v=0$.

We obtain a $144 \times 144$ quartic pencil, whose 576 eigenvalues are shown in Figure 1 given above.

$$
\text { err }=\sum_{i=1}^{576}\left\|P\left(\lambda_{i}\right) v^{(i)}\right\|_{2}=1.2471 e-014
$$

Example 2. [10]

Now, let us consider the following quadratic eigenvalue problems given by $\left(\lambda^{2} I_{n}+\lambda C+K\right) v=0$. The 400 eigenvalues are shown in Figure 2 below

$$
\text { err }=\sum_{i=1}^{400}\left\|P\left(\lambda_{i}\right) v^{(i)}\right\|_{2}=3.1581 e-013
$$

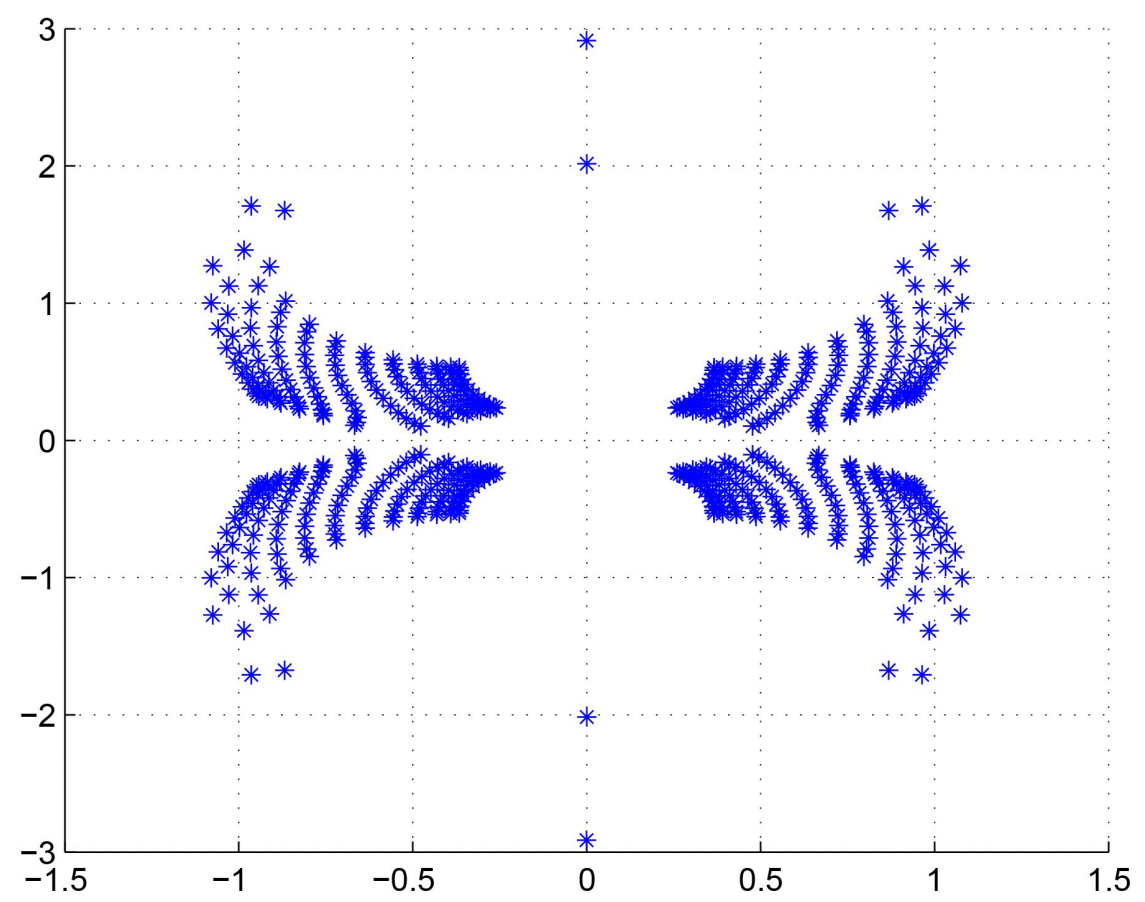

Figure 1. Eigenvalues of $144 \times 144$ polynomial problems. 


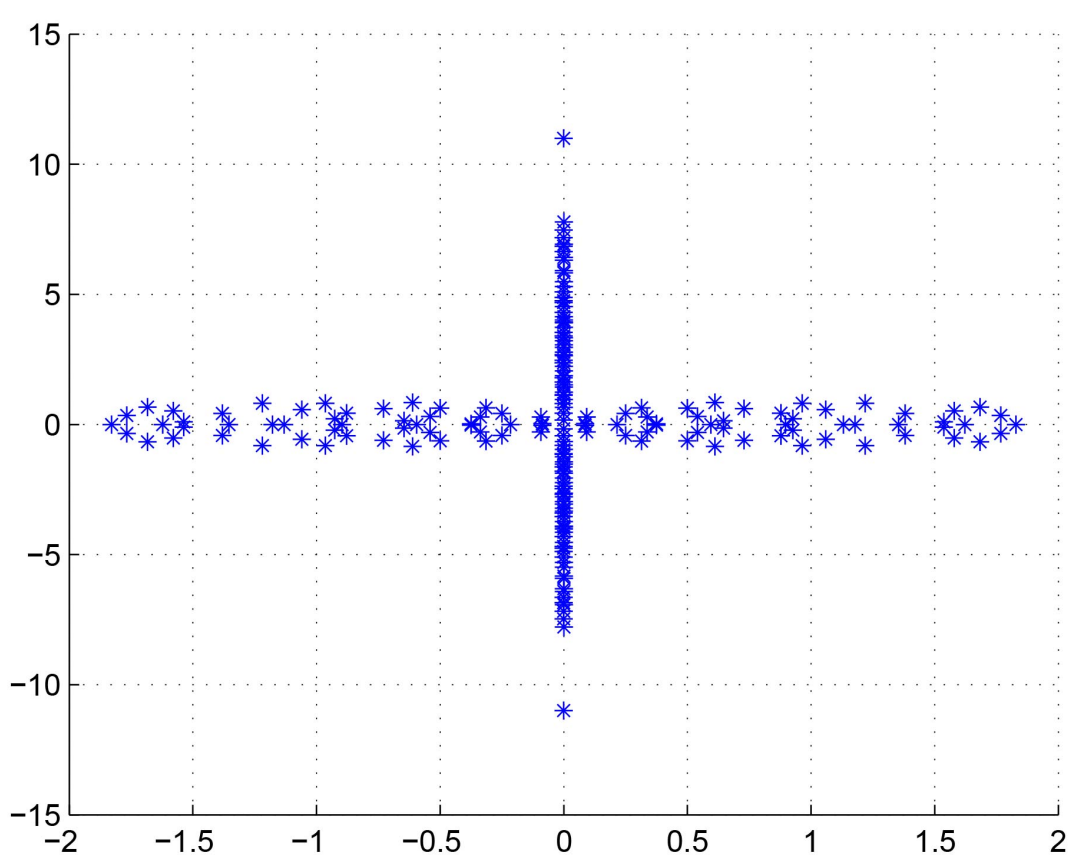

Figure 2. Eigenvalues of 400 quadratic polynomial problems.

\section{Conclusion}

We have proposed a numerical approach for solving polynomial eigenvalue problems structured. We first transform polynomial eigenvalue problem $P(\lambda) v=\sum_{i=0}^{k} \lambda^{i} M_{i} v=0$ to a skew-Hamiltonian/Hamiltonian pencil $(A-\lambda B) v=0$. The second step is to transform the pencil into a standard Hamiltonian eigenproblem $\mathrm{Hz}=\lambda \mathrm{z}$. Numerical methods based on these structured linearizations are expected to be more effective in computing accurate eigenvalues in practical applications. My future work based on this current study is to solve the large matrix equations applied in signal processing, image restoration and model reduction in control theory.

\section{Acknowledgements}

We thank the editor and the referee for their comments.

\section{Conflicts of Interest}

The author declares no conflicts of interest regarding the publication of this paper.

\section{References}

[1] Bora, S., Karow, M., Mehl, C. and Sharma, P. (2014) Structured Eigenvalue Backward Errors of Matrix Pencils and Polynomials with Hermitian and Related Structures. SIAM Journal on Matrix Analysis and Applications, 35, 453-475. https://doi.org/10.1137/130925621

[2] Higham, N.J. (2005) Solving Polynomial Eigenproblems by Linearization. School of Mathematics, The University of Manchester, Manchester, M60 1QD. 
[3] Lu, Z.J., van der Vegt, J.J.W. and Xu, Y. (2018) Spectral Approximation for Polynomial Eigenvalue Problems. Computers and Mathematics with Applications, 76, 1184-1197. https://doi.org/10.1016/j.camwa.2018.06.007

[4] Mehrmann, V. and Watkins, D. (2000) Structure-Preserving Methods for Computing Eigenpairs of Large Sparse Skew-Hamiltonian/Hamiltonian Pencils. SIAM Journal on Scientific Computing, 22, 1905-1925.

https://doi.org/10.1137/S1064827500366434

[5] Perovia, V. and Mackey, D.S. (2018) Linearizations of Matrix Polynomials in Newton Bases. Linear Algebra and Its Applications, 556, 1-45. https://doi.org/10.1016/j.laa.2018.06.030

[6] Benner, P., Byers, R., Fassbender, H., Mehrmann, V. and Watkins, D. (2000) Cholesky-Like Factorizations of Skew-Symmetric Matrices. Electronic Transactions on Numerical Analysis, 11, 85-93.

[7] Fassbender, H., Mackey, D.S., Mackey, N. and Xu, H.G. (1998) Hamiltonian Square Roots of Skew-Hamiltonian Matrices. Linear Algebra and Its Applications, 287, 125-159. https://doi.org/10.1016/S0024-3795(98)10137-4

[8] Mackey, D.S., Mackey, N., Mehl, C. and Mehrmann, V. (2005) Structured Polynomial Eigenvalue Problems: Good Vibrations from Good Linearizations. Technical Report 239, DFG Research Center Matheon, Mathematics for Key Technologies, Berlin.

[9] Mehrmann, V. and Watkins, D. (2002) Polynomial Eigenvalue Problems with Hamiltonian Structure. Electronic Transactions on Numerical Analysis, 13, 106-118.

[10] Fassbender, H. and Kressner, D. (2006) Structured Eigenvalue Problems. GAMM-Mitteilungen, 29, 297-318. https://doi.org/10.1002/gamm.201490035 